\title{
Optimal control strategy for prevention of avian influenza pandemic
}

\author{
Eunok Jung ${ }^{a}$, Shingo Iwami ${ }^{b}{ }^{*}$, Yasuhiro Takeuchi ${ }^{b}$, Tae-Chang Jo ${ }^{c}$ \\ Department of Mathematics, Konkuk University, Korea ${ }^{a}$, \\ Graduate School of Science and Technology, Shizuoka University, Japan ${ }^{b}$, \\ Department of Mathematics, Inha University, Korea ${ }^{c}$
}

\begin{abstract}
The spread of H5N1 virus to Europe and continued human infection in Southeast Asia have heightened pandemic concern. Although, fortunately, sustained human-to-human transmissions have not been reported yet, it is said that a pandemic virus which can be easily transmitted among humans certainly emerges in the future. In this study, we extended the previous studies for the prevention of the pandemic influenza to evaluate the timedependent optimal prevention policies, which are associated with elimination policy and quarantine policy, considering its execution cost. Actually, the execution cost affects the optimal strategy of prevention policies and the prevention of the disease spread. We found that the quarantine policy is very important rather than the elimination policy during the disease spread, even if the unit execution cost of the quarantine policy is more expensive than that of the elimination policy. And also, the change of the unit execution cost does affect the total cumulative cost of the optimal prevention policies but does not affect the relative frequency of each cumulative execution cost. Furthermore, interestingly, we revealed that an optimal strategy to reduce the number of total infected humans might increase a chance of invadability of the mutant influenza.
\end{abstract}

Keywords: Epidemic model; Avian influenza; Optimal control theory; Elimination policy; Quarantine policy; Invadability

${ }^{*}$ To whom correspondence should be addressed. E-mail: yukitadahara@yahoo.co.jp 


\section{Introduction}

Preparedness for a possible influenza pandemic caused by highly pathogenic avian influenza A subtype H5N1 has become a global priority $[40,42,48]$. The spread of the virus to Europe and continued human infection in Southeast Asia have heightened pandemic concern [5]. Although, fortunately, sustained human-to-human transmissions have not been reported yet (except for a few cases caused by close contacts within household $[26,35,40]$ ), it is said that a pandemic virus which can be easily transmitted among humans (i.e., mutant avian influenza) certainly emerges in the future $[12,35,40]$. Therefore, the WHO recommends that all countries undertake urgent action to prepare for a pandemic influenza.

The US General Accounting Office estimated the human consequences of a pandemic of influenza in the US. These consequences includes 200,000,000 Americans infected, 90,000,000 clinically ill and 2,000,000 dead. The study also estimates that $30 \%$ of all US workers would become ill and $2.5 \%$ would die, with $30 \%$ of workers missing a mean of 3 weeks of work, and a subsequent decrease in the US Gross domestic product of 5\% [35]. Thus, influenza produces several costs due to lost productivity and associated medical treatment, as well as execution costs of preventative policies $[14,35,38]$. Actually, in the US, influenza is responsible for a total cost of over 10 billion per year and further it has been estimated that a future pandemic could cause hundreds of billions of dollars in the above costs [32]. In particular, the costs associated with vaccination, elimination of birds, hospitalization, quarantine of persons, transport restriction and so on can be great.

To project the potential economic impact of pandemic influenza mitigation strategies, we consider optimal control strategies associated with elimination policy and quarantine policy including execution costs based on previous model in $[15,16,17]$. The optimal control theory, which was developed by Pontryagin and his co-workers in the late 1950s, has been applied to many areas including economics, management, engineering, biology, physiology, and medicine [10, 18, 19, 20, 22, 24]. We introduce two control mechanisms in the model: one is the effort of the elimination policy for infected birds and the other is the effort of the quarantine policy for infected humans. In our system, the elimination control is manipulated in the term of the force of infection by infected birds with wild avian influenza and the quarantine control is manipulated in the term of the force of infection by infected humans with mutant avian influenza. We found that the quarantine policy is very important rather than the elimination policy during the disease spread, even if the unit execution cost of the quarantine policy is more expensive than that of the elimination policy. And 
also, the change of the unit execution cost does affect the total cumulative cost of the optimal prevention policies but does not affect the relative frequency of each cumulative execution cost. Furthermore, interestingly, we revealed that an optimal strategy to reduce the number of total infected humans might increase a chance of invadability of the mutant influenza.

\section{Materials and Methods}

Recent outbreaks of emerging infectious diseases such as SARS and H5N1 avian influenza have underlined the fact that the infectious disease of wild animals might acquire the ability to spread efficiently among the humans [2]. Therefore, in order to capture an essence of invasion and transmission of avian influenza, we consider both bird and human worlds.

\subsection{Mathematical model}

Herein, we describe a combination model with avian influenza transmission dynamics among birds and humans including execution costs of time-dependent elimination and quarantine policy after the occurrence of mutant avian influenza. Our mathematical model is given by the following equations:

$$
\begin{aligned}
& X^{\prime}=c-b X-\left(1-u_{1}(t)\right) \omega X Y, \\
& Y^{\prime}=\left(1-u_{1}(t)\right) \omega X Y-(b+m) Y, \\
& S^{\prime}=\lambda-\mu S-\beta_{1} Y S-\left(1-u_{2}(t)\right) \beta_{2} H S, \\
& B^{\prime}=\beta_{1} S Y-\left(\mu+d_{1}\right) B \\
& H^{\prime}=\left(1-u_{2}(t)\right) \beta_{2} S H-\left(\mu+d_{2}\right) H .
\end{aligned}
$$

All birds and humans in the effective population are divided into several compartments, respectively including susceptible birds $(X)$, birds infected with wild avian influenza $(Y)$, susceptible humans $(S)$, humans infected with wild avian influenza $(B)$, and humans infected with mutant avian influenza $(H)$. The parameters $c$ and $\lambda$ are the rates of birth for birds and humans, respectively. Birds and humans die from the natural causes at the rates $b$ and $\mu$, respectively. Furthermore, $m$ and $d_{1}$ are the death rates inflicted by wild avian influenza and $d_{2}$ is the additional death rate induced by mutant avian influenza (i.e., $d_{1}$ and $d_{2}$, respectively, represent a virulence of the wild and mutant strain). Here we mention that the effect of recovery from the infection is not considered (i.e., we assume that the infection is fatal) because we are interested in the worst situation of the pandemic (in [17], we consider the 
effect of recovery in detail). The parameters $\omega$ and $\beta_{1}$ are the rate at which wild avian influenza is contracted from an average infected bird, $\beta_{2}$ is the transmission rate of mutant avian influenza among humans.

We remark that the transmission of wild avian influenza for humans is restricted from birds to humans (wild avian influenza is just transmitted directly from birds to humans $[4,43,44,50])$ but mutant avian influenza can be transmitted among humans. Actually, the mutant avian influenza has not emerged yet but many experts expect the emergence, and therefore, we consider the situation after the emergence of the mutant (see $[16,17]$ for more detailed explanation).

Here the control functions, $u_{1}(t)$ and $u_{2}(t)$, are bounded, Lebesgue integrable functions. The coefficient with "elimination control", $u_{1}(t)$, is the effort to reduce the number of infected birds (we simply assume that a decrease of $\omega$ leads to reducing the number of infected birds because we obtain qualitatively similar results even if the elimination control is modeled by different way). The "quarantine control", $u_{2}(t)$, represents the effort to reduce the number of contacts with humans infected with mutant avian influenza. For example, when the quarantine control $u_{2}$ is large, there is low infective contacts rate and high implementation costs. Note that the controls, $u_{1}(t)$ is bounded in $[0,1]$ and $u_{2}(t)$ is bounded in $[0, a]$, where $0<a<1$. The upper bound $a$ is determined by the basic reproduction number of mutant avian influenza $R_{0}$ (see Epidemiological scenarios).

In $[15,16,17]$, we investigated the effect of time-independent prevention policies at the final phase of mutant avian influenza spread (i.e., equilibrium state) without an execution cost. In this paper, we investigate how we should time-dependently execute the prevention policies in order to minimize the number of total infected humans $(B(t)+H(t))$ keeping total cost of the policies (cumulated values of execution costs of both policies for total days) low during the spread. The time-dependent optimal prevention policies can be obtained by minimizing the following objective functional:

$$
J\left(u_{1}, u_{2}\right)=\int_{0}^{t_{f}}\left[B(t)+H(t)+\frac{B_{1}}{2} u_{1}^{2}(t)+\frac{B_{2}}{2} u_{2}^{2}(t)\right] d t .
$$

The costs of the elimination and quarantine policies are nonlinear and take quadratic forms. Here the coefficients $B_{1}$ and $B_{2}$, respectively, represent balancing cost factors due to size and importance of the other three parts of the objective functional. Therefore, for example, large (small) values of $B_{1}$ and $B_{2}$ imply expensive (cheap) unit execution costs of elimination and quarantine policies because $B_{1} u_{1}^{2} / 2$ and $B_{2} u_{2}^{2} / 2$ become large (small). 
Our goal is to find an optimal control pair, $u_{1}^{*}(t)$ and $u_{2}^{*}(t)$, such that

$$
J\left(u_{1}^{*}, u_{2}^{*}\right)=\min _{\Omega} J\left(u_{1}, u_{2}\right),
$$

where $\Omega=\left\{\left(u_{1}, u_{2}\right) \in L^{1}\left(0, t_{f}\right) \mid 0 \leq u_{1} \leq 1,0 \leq u_{2} \leq a\right\}$. We use Pontryagin's Maximum Principle [21, 39] to solve the optimal control problem and fix $t_{f}=365$. The analysis of the optimal control problem is referred to Appendix B.

\subsection{Basic reproduction numbers}

A measure of transmissibility and of the stringency of control policies required to stop an epidemic is the basic reproduction number, which is the number of secondary cases produced by each primary case [1]. We obtain two basic reproduction numbers, $r_{0}$ for infected bird with wild avian influenza and $R_{0}$ for infected human with mutant avian influenza, by model (1) without the prevention policies (i.e., $u_{1}=u_{2}=0$ ). Here we define

$$
r_{0}=\frac{\omega}{b+m} \frac{c}{b}, \quad R_{0}=\frac{\beta_{2}}{\mu+d_{2}} \frac{\lambda}{\mu} .
$$

However, for mutant avian influenza we are unable to describe adequately the spread of the infection by applying these usual basic reproduction numbers. The reason is that avian influenza is already endemic, particularly in Asian poultry [3, 46, 49]. This implies that some portion of humans have already been infected with wild avian influenza and are dead (and some portion have cross-immunity to mutant avian influenza in the extended model which includes a possibility of recovery) after avian influenza is endemic among birds (note that, in the case of basic reproduction numbers, all humans are susceptible). We can define an invasion reproduction number for the mutant strain of avian influenza,

$$
\bar{R}_{0}=\frac{\beta_{2}}{\mu+d_{2}} \frac{\lambda}{\mu+\beta_{1}(c /(b+m)-b / \omega)},
$$

which means an expected number of new infectious cases after avian influenza becomes endemic among birds (i.e., after $r_{0}$ becomes larger than 1 ). Thus, $\bar{R}_{0}$ can capture the real situations and the essence of the spread of mutant avian influenza.

The dynamical behavior of our model are completely classified by these basic and invasion reproduction numbers. The detailed explanations are given in Appendix A.

\subsection{Estimation of epidemiological parameters}

Baseline values of model parameters used for simulations are presented in Table 1. These parameters are based on previous epidemics among birds (the H5N1 2004 
epidemic $[13,43,44])$ and influenza pandemics among humans (the H1N1 1918-1919 pandemic $[6,7,27,30,36,47])$.

Estimated basic reproduction numbers of infected birds during the 2004 epidemic range from 1.71 to 5.00 [44]. Here we use a relatively small basic reproduction number $r_{0}=1.86$. An initial bird population size is assumed to be $c / b=5.00 \times 10^{2}$ individuals. Usually mean lifespan of poultry is about 2 years, but we consider that the mean duration of bird being in effective population is about $1 / b=100$ days because of migration or marketing. Therefore, birth or restocking rate of birds are $c=5$ individuals per day. Because estimated mean infectious periods of infected birds range from 5 to $17.6[29,41,44]$, we assume that the mean infectious period is about $1 / m=10$ days (i.e., $m=0.10$ day $^{-1}$ ). Now we can estimate that transmission rate of the wild strain among birds is $\omega=4.10 \times 10^{-4}$ day $^{-1}$ individual $^{-1}$.

On the other hand, well-known estimated 1918-1919 pandemic basic reproduction numbers vary very widely, ranging from 1.2 to $20[6,7,27,30,36,47]$. Here the basic reproduction number is assumed to be $R_{0}=3.79$ which is relatively large. An initial human population size is set in $\lambda / \mu=1.00 \times 10^{3}$ individuals. We assume that mean duration of humans being in the effective population is about $1 / \mu=365$ days (mean lifespan of human is about 60 years) because of rapid human migration, transportation and so on. This implies that birth or immigration rate of humans are $\lambda=2.7$ individuals per day. And also, it is considered that estimated mean infectious period of infected humans with wild avian influenza are distributed on range from 6 to 30 days $[23,50]$. However, the expected virulence of a mutant of H5N1 causing pandemic is uncertain, which means that the mutant strain may be more or less virulent than the wild strain $[1,4]$. Therefore we have to consider the situations of both higher and lower virulence cases. We assume that the mean infectious period of wild and mutant avian influenza are, respectively, $1 / d_{1}=10$ days and $1 / d_{2}=14$ days for "lower virulence case $\left(d_{1}>d_{2}\right)$ " (the virulence of wild and mutant avian influenza virus are $d_{1}=0.1 \mathrm{day}^{-1}$ and $d_{2}=0.07 \mathrm{day}^{-1}$ ). In addition, we consider $d_{1}=0.04$ day $^{-1}$ as a "higher virulence case $\left(d_{1}<d_{2}\right)$ ". Now we can estimate that transmission parameter for the mutant strain among humans is $\beta_{2}=2.80 \times 10^{-4}$ day $^{-1}$ individual $^{-1}$. Furthermore, we assume that the transmission rate of the wild strain among humans (i.e., from infected birds to susceptible humans: the transmission rate between species, in general, seems to be smaller than one within species) is $\beta_{1}=2.00 \times 10^{-4}$ day $^{-1}$ individual $^{-1}$. 


\subsection{Epidemiological setting}

We have mentioned that wild avian influenza is already endemic among birds [3, 46, 49]. Therefore, we must conclude that, unless a policy aimed at reducing $r_{0}$ is applied, $r_{0}>1$ holds among birds. On the other hand, although there is no evidence of sustained human-to-human transmission yet [26, 50], we have to consider the situation that mutant avian influenza can effectively spread. Therefore, we have to conclude that, unless a policy aimed at reducing $\bar{R}_{0}$ is applied, $\bar{R}_{0}>1$ holds among humans. Thus we are interested in and assume the case (iv) in Theorem $A 2$ to apply optimal control theory (note that our baseline parameter values are satisfied with the case $(i v)$ ). Furthermore, because some portion of birds and humans have already been infected with wild avian influenza or are dead, we assume that $X(0)=268$, $Y(0)=21, S(0)=397, B(0)=16$ and $H(0)=1$ (these values are chosen from near the steady state, for the appropriate parameter values in Table 1 , that wild avian influenza has been spread through the bird and human population (i.e., $E_{*}$ in Appendix A)).

\subsection{Control setting}

During the optimal of prevention policies, the basic reproduction numbers can be rewritten as follows:

$$
r_{0}^{*}(t)=\frac{\omega\left(1-u_{1}^{*}(t)\right)}{b+m} \frac{c}{b}, \quad R_{0}^{*}(t)=\frac{\beta_{2}\left(1-u_{2}^{*}(t)\right)}{\mu+d_{2}} \frac{\lambda}{\mu} .
$$

The optimal elimination and quarantine policies, $u_{1}^{*}(t)$ and $u_{2}^{*}(t)$, decrease the value of $r_{0}^{*}(t)$ and $R_{0}^{*}(t)$, respectively. However, in the real life situation, we can hardly expect that the quarantine policy reduces $R_{0}^{*}(t)$ to the value less than 1 (although the elimination policy is relatively easy to execute and has succeeded in some regions $[41,45,51])$. Therefore, we assume that, for the quarantine policy, the reduced value still satisfies $R_{0}^{*}(t)>1$, which implies that

$$
R_{0}^{*}(t)>1 \Longleftrightarrow u_{2}^{*}(t)<1-\frac{1}{R_{0}}=a .
$$

Thus, we set the upper bound $a$ of control function $u_{2}$ (since $R_{0}=3.79$ in Table 1 , we can determine the upper bound $a=0.74)$. Actually, if $R_{0}^{*}(t)<1$ could be realized, the optimal policies effectively prevent the disease spread (simulations are not shown). And also, we assume the balancing factor $B_{2}$ associated with control $u_{2}$ is bigger than $B_{1}$ associated with a control $u_{1}$ because to restrict human activity is more difficult than to eliminate infected birds. Although the weight of unit execution 
cost might be different on the situation, we fixed that $\left(B_{1}, B_{2}\right)=(0.1,1)$ and $(1,10)$ as default values of our control problem (we preserve $B_{2}$ is order 10 greater than $B_{1}$ in other cost situations).

\section{Results}

The optimal strategy is obtained by solving the state and adjoint systems and the optimality equations. An iterative method is used for solving the optimality system as follows: First, we start to solve the state equations (1) with a guess for the controls over the simulated time. Second, using the current iteration solution of the state equations, the adjoint equations are solved by a backward method with the transversality conditions (7) in Appendix B. Then, update the controls by using a convex combination of the controls in the previous iteration and the value from the characterizations (8), in Appendix B, in the current iteration. Repeat this process and stop iterations if the values of unknowns at the previous iteration are very close to the ones at the present iteration. For detailed description of the iterative method, see Chapter 4 and 8.2 in the reference [25].

\subsection{Optimal strategy of prevention policies}

We investigate the time-course of disease spread with optimal strategy of prevention policies for 365 days after the emergence of mutant avian influenza virus. The dashed black curves of the top figures in Fig.1 and 2 represent the epidemic curves of total infected humans without any policy in the lower and higher virulence cases, respectively. We can see that mutant avian influenza leads to a pandemic of the disease in the both cases.

The colored curves (the blue and red curves correspond to $\left(B_{1}, B_{2}\right)=(0.1,1)$ and $(1,10)$, respectively) of the top figures in Fig. 1 and 2 represent the epidemic curves of total infected humans with optimal prevention policies in the lower and higher virulence cases, respectively. If we can execute the optimal strategy, the epidemic curves are dramatically reduced in the both cases.

The bottom figures of Fig.1 and 2 show the time-dependent optimal strategy of prevention policies $u_{1}^{*}(t)$ and $u_{2}^{*}(t)$ in the lower and higher virulence cases, respectively. In the both virulence case, the short intensive effort of the elimination policy $u_{1}^{*}(t)$ is needed at the beginning of the disease spread and then the effort is smoothly reduced. On the other hand, the long strong effort of the quarantine policy $u_{2}^{*}(t)$ is needed over the simulated time. Thus, in spite of the fact that the unit cost 
of the quarantine policy is 10 times as expensive as that of the elimination policy, actually, $u_{2}^{*}(t)$ remains at high level for a long time than $u_{1}^{*}(t)$. Therefore, the optimal strategy shows that the quarantine policy is more important for the lower and higher virulence cases. These conclusions are partially different when we consider a time-independent optimal strategy of prevention policies at the final phase of the disease spread in $[15,16,17]$ (see Discussion).

\subsection{Cost of prevention policies}

We investigate an impact of the unit execution cost of prevention policies on the optimal strategy (see the blue and red curves in the bottom figures of Fig.1 and 2 ). As the unit execution cost increases, in general, the effort of prevention policies decreases over the simulated time. And also, if the unit execution cost is extremely expensive, then the optimal policies no longer reduce the epidemic curve and can not prevent the disease spread (simulations are not shown). Thus, the unit execution cost affects the optimal strategy of prevention policies and the prevention of the disease spread.

Furthermore, we reveal relations between the unit execution cost and the cumulative execution cost of the optimal prevention policies. Define the cumulative execution cost of the elimination $\left(T_{e}\right)$ and quarantine $\left(T_{q}\right)$ policies are, respectively,

$$
T_{e}=\frac{1}{2} \int_{0}^{t_{f}} B_{1} u_{1}^{*}(t)^{2} d t, \quad T_{q}=\frac{1}{2} \int_{0}^{t_{f}} B_{2} u_{2}^{*}(t)^{2} d t .
$$

We calculate each cumulative execution cost for the case $\left(B_{1}, B_{2}\right)=(0.05,0.5)$, $(0.1,1),(1,10)$ and $(5,50)$ in the lower virulence case in Fig.3 (these unit execution costs are relatively cheap, and therefore the disease spread are comparatively suppressed by the optimal strategy). The top and bottom figures of Fig.3, respectively, represent the total cumulative cost and the relative frequency of each cumulative execution cost. The pink and green bars correspond to the cumulative cost of the elimination and quarantine policies, respectively. Here we do not consider the higher virulence case but the conclusion for this case is qualitatively the same.

The total cumulative cost $\left(T_{e}+T_{q}\right)$ increases but is not proportion to the unit execution cost as the unit cost increases (the top figure). It shows that the total cost of using the quarantine policy is much expensive than one of using the elimination policy. On the other hand, the relative frequency of each cumulative execution cost $\left(T_{e}: T_{q}\right)$ is almost same even if the unit execution cost increases (the bottom figure). Thus, the change of the unit execution cost does affect the total cumulative cost but does not affect the relative frequency of each cumulative execution cost. This 
implies that the relative cost of using the quarantine policy is much expensive than one of using the elimination policy in the optimal strategies.

\subsection{Invadability of mutant avian influenza}

We investigate how the optimal strategy affects the invadability of mutant avian influenza. Define the invadability measure of mutant avian influenza strain $\bar{R}_{0}^{*}(t)$ as follows. If $r_{0}<1$, the wild strain can not spread. The invadability is only determined by the basic reproduction number of the mutant strain $R_{0}$ (see Theorem $A$ 2. (i) and (ii)). Therefore, if $r_{0}^{*}(t)<1$, then $\bar{R}_{0}^{*}(t)=R_{0}^{*}(t)$. However, if $r_{0}>1$, the wild strain spreads. Then we use the invasion reproduction number of the mutant strain $\bar{R}_{0}$ to investigate whether the mutant strain spreads (see Theorem A 2. (iii) and (iv)). Therefore, if $r_{0}^{*}(t)>1$, then

$$
\bar{R}_{0}^{*}(t)=\frac{\beta_{2}\left(1-u_{2}^{*}(t)\right)}{\mu+d_{2}} \frac{\lambda}{\mu+\beta_{1}\left(\frac{c}{b+m}-\frac{b}{\omega\left(1-u_{1}^{*}(t)\right)}\right)} .
$$

Thus, if the invadability measure $\bar{R}_{0}^{*}(t)$ is less than 1 , then the mutant strain does not spread among humans irrespective of $r_{0}^{*}(t)$ : otherwise, the mutant strain spreads. Therefore, the value of the invadability measure represents a magnitude of the spread of mutant avian influenza (i.e, the spread of mutant avian influenza weakens as the invadability measure decreases even if $\left.\bar{R}_{0}^{*}(t)>1\right)$.

We calculate the invadability measure of the mutant strain $\bar{R}_{0}^{*}(t)$ in Fig.4 during the optimal prevention policies for the unit execution cost $\left(B_{1}, B_{2}\right)=(0.1,1)$ and $(1,10)$ in the lower virulence case (the conclusion for the higher virulence case is qualitatively the same). The optimal strategy suppresses the spread of the mutant strain because the invadability measure is reduced less than $\bar{R}_{0}^{*}(0)=1.5$ (which is the value of the invadability measure before execution of the optimal prevention policies: see Table 1) at the beginning. In general, as the unit execution cost increases, the invadability measure increases over the simulated time (see the blue and red curves in Fig.4). Therefore, a cheap unit execution cost effectively reduces the magnitude of the spread of mutant avian influenza. Furthermore, interestingly, we can see that the invadability measure gradually increases at the beginning of the optimal prevention policies and has a peak in both cases. This implies that the optimal strategy to reduce the number of total infected humans might increase a chance of invadability of mutant avian influenza. The counter-intuitive result is caused by the competition between the wild and mutant strain. Actually, because the unit execution cost of the elimination policy is cheaper than one of the quarantine policy in order to 
reduce the total number, the effort of the elimination policy is large and one of the quarantine policy is small at the beginning (see Fig.1). Therefore, the basic reproductive number $r_{0}^{*}(t)$ decreases (simulations are not shown), the mutant strain

obtains some competitive advantage, and the invadability measure $\bar{R}_{0}^{*}(t)$ increases. Thus to eliminate the infected birds might increase the potential invasiveness of the mutant strain among humans. Hence the optimal strategy is no longer optimum in terms of prevention of invadability of mutant avian influenza.

\section{Discussion}

Most of economic studies on avian influenza have so far concentrated on the impact of the disease, more studies are needed to assess the impact of its control strategy in term of sustainability and efficacy [33, 37]. Epidemiological models including economic aspects are needed to estimate impacts of execution costs of prevention policies [33]. Actually, even mitigating programs which execute the same intervention policies have different unit execution costs depending on various situations $[28,31,32]$. In this study, we extended the previous studies for the prevention of mutant avian influenza spread to evaluate the time-dependent optimal prevention policies with considering its execution cost. Here we assumed that the unit execution cost is relatively low. Our findings demonstrated that the optimal policies can dramatically reduce the pandemic phase and block the population wide spread of the disease (the top figures of Fig.1 and 2). And also, we showed that the quarantine policy is very important rather than the elimination policy during the optimal prevention policies, even if the unit execution cost of the quarantine policy is more expensive than that of the elimination policy in the both virulence cases (the bottom figures in Fig.1 and 2). This result is partially different from our conclusions obtained in $[16,17]$ because we previously concluded that the quarantine policy might increase the total number of infected humans at the equilibrium in the higher virulence case when we time-independently investigated the efficacy of the elimination and quarantine policies. Furthermore, we found that the unit execution cost affects the optimal strategy of prevention policies because the effort of prevention policies decreases as the unit execution cost increases (the bottom figures in Fig.1 and 2). However, interestingly, the increase of the unit execution cost affects the total cumulative cost but does not affect the relative frequency of each cumulative execution cost (Fig.3). This suggests that the relative cost of using the quarantine policy does not change. Another important result obtained here is that the optimal strategy to reduce the number of total infected humans is no longer optimum in 
terms of prevention of invadability of mutant avian influenza (Fig.4). We revealed that the invadability measure gradually increases at the beginning of the optimal prevention policies. This implies that the optimal prevention policies are not robust for an influx of the infected humans with the mutant strain (i.e, the optimal policies are sensitive to pertubations). Therefore, to succeed the optimal prevention policy needs restriction of movement and screening of the infected humans. Thus, our work highlights that to consider the transient dynamics and the execution cost of prevention policies is critically important to develop effective interventions against next influenza pandemic.

\section{Appendix A: Dynamical behavior}

We consider system (1) without controls (i.e., $u_{1}(t)$ and $u_{2}(t)$ are set to be zero). Because the behavior of $X$ and $Y$ of system (1) is independent of the other dynamics, we obtain the following properties of the behavior. The proof of this theorem is referred to Theorem 3.1 in [15].

Theorem A 1. If $r_{0} \leq 1$, then $X$ and $Y$ converge to $X_{0}$ and 0 , respectively, as $t \rightarrow \infty$. On the other hand if $r_{0}>1$, then $X$ and $Y$ converge to $X_{+}$and $Y_{+}$, respectively, where $X_{0}=c / b, X_{+}=(b+m) / \omega$ and $Y_{+}=c /(b+m)-b / \omega$.

We can neglect the dynamics of $Y(t)$ in system (1) because it is completely determined by Theorem $A$. Then (1) can be reduced to the following model:

$$
\begin{aligned}
& S^{\prime}=\lambda-\mu S-\left(\beta_{1} \tilde{Y}+\beta_{2} H\right) S \\
& B^{\prime}=\beta_{1} S \tilde{Y}-\left(\mu+d_{1}\right) B \\
& H^{\prime}=\beta_{2} S H-\left(\mu+d_{2}\right) H .
\end{aligned}
$$

Here we remark that $\tilde{Y}=0$ or $Y_{+}$. This system has four equilibrium states:

$E_{0}=\left(S_{0}, 0,0\right)$, where $S_{0}=\frac{\lambda}{\mu}$;

$E_{b}=\left(S_{b}, 0, H_{b}\right)$, where $S_{b}=\frac{\mu+d_{2}}{\beta_{2}}, H_{b}=\frac{\lambda}{\mu+d_{2}}-\frac{\mu}{\beta_{2}} ;$

$E_{*}=\left(S_{*}, B_{*}, 0\right), \quad$ where $S_{*}=\frac{\lambda}{\mu+\beta_{1} Y_{+}}, B_{*}=\frac{\beta_{1} Y_{+} S_{*}}{\mu+d_{1}} ;$

$E_{+}=\left(S_{+}, B_{+}, H_{+}\right)$, where $S_{+}=\frac{\mu+d_{2}}{\beta_{2}}, B_{+}=\frac{\beta_{1} Y_{+} S_{+}}{\mu+d_{1}}, H_{+}=\frac{\lambda-\mu S_{+}-\beta_{1} Y_{+} S_{+}}{\beta_{2} S_{+}}$.

The properties of system (4) are given by the following theorem: 
Theorem A 2. (i) If $r_{0} \leq 1$ and $R_{0} \leq 1$, then $E_{0}$ is globally asymptotically stable (GAS) which means that the orbit converges to the equilibrium as $t \rightarrow \infty$ for arbitrary initial point.

(ii) If $r_{0} \leq 1$ and $R_{0}>1$, then $E_{b}$ is $G A S$.

(iii) If $r_{0}>1$ and $\bar{R}_{0} \leq 1$, then $E_{*}$ is $G A S$.

(iv) If $r_{0}>1$ and $\bar{R}_{0}>1$, then $E_{+}$is GAS.

The proof of this theorem is referred to Theorem B.1 in [16].

\section{Appendix B: Analysis of optimal control problem}

We apply Pontryagin's Maximum Principle $[21,39]$ to the constrained control problem, then this principle converts (1) - (3) into a problem of minimizing pointwise a Lagrangian, $L$, with respect to $u_{1}$ and $u_{2}$. The Lagrangian for our problem is the integrand of the objective functional coupled with the five right hand sides of the state equations.

$$
L\left(X, Y, S, B, H, u_{1}, u_{2}, \lambda_{1}, \lambda_{2}, \lambda_{3}, \lambda_{4}, \lambda_{5}\right)=B(t)+H(t)+\frac{B_{1}}{2} u_{1}^{2}+\frac{B_{2}}{2} u_{2}^{2}+\sum_{i=1}^{5} \lambda_{i} g_{i},
$$

where $g_{i}$ is the right hand side of the differential equation of the $i$ th state variable. By applying Pontryagin's Maximum Principle [34] and the existence result for the optimal control pairs from [11], we obtain the following theorem.

Theorem B 1. There exists an optimal control pair $u_{1}^{*}, u_{2}^{*}$ and corresponding solution, $X^{*}, Y^{*}, S^{*}, B^{*}$, and $H^{*}$, that minimizes $J\left(u_{1}, u_{2}\right)$ over $\Omega$. Furthermore, there exists adjoint functions, $\lambda_{1}(t), \ldots, \lambda_{5}(t)$, such that

$$
\begin{aligned}
& \dot{\lambda}_{1}=\lambda_{1}\left(b+\left(1-u_{1}(t)\right) \omega Y\right)+\lambda_{2}\left(-\omega\left(1-u_{1}(t)\right) Y\right), \\
& \dot{\lambda}_{2}=\lambda_{1}\left(\omega\left(1-u_{1}(t)\right) X\right)+\lambda_{2}\left(-\omega\left(1-u_{1}(t)\right) X+(b+m)\right)+\lambda_{3}\left(\beta_{1} S\right)+\lambda_{4}\left(-\beta_{1} S\right), \\
& \dot{\lambda}_{3}=\lambda_{3}\left(\mu+\beta_{1} Y+\beta_{2}\left(1-u_{2}(t)\right) H\right)+\lambda_{4}\left(-\beta_{1} Y\right)+\lambda_{5}\left(-\beta_{2}\left(1-u_{2}(t)\right) H\right), \\
& \dot{\lambda}_{4}=-1+\lambda_{4}\left(\mu+d_{1}\right), \\
& \dot{\lambda}_{5}=-1+\lambda_{3}\left(\beta_{2}\left(1-u_{2}(t)\right) S\right)+\lambda_{5}\left(-\beta_{2}\left(1-u_{2}\right) S+\mu+d_{2}\right),
\end{aligned}
$$

with transversality conditions

$$
\lambda_{i}\left(t_{f}\right)=0, i=1, \ldots, 5
$$


The following characterization holds

$$
\begin{aligned}
& u_{1}^{*}(t)=\min \left(\max \left(0, \frac{1}{B_{1}}\left(\lambda_{2}-\lambda_{1}\right) \omega X^{*} Y^{*}\right), 1\right) \\
& \text { and } \\
& u_{2}^{*}(t)=\min \left(\max \left(0, \frac{1}{B_{2}}\left(\lambda_{5}-\lambda_{3}\right) \beta_{2} S^{*} H^{*}\right), a\right) .
\end{aligned}
$$

Proof. The existence of an optimal control pair is given in Corollary 4.1 of [11]. In our problem, the convexity of integrand of $J$ with respect to $\left(u_{1}, u_{2}\right)$, a priori boundedness of the state solutions, and the Lipschitz property of the state system with respect to the state variables show the existence of optimal solutions in our optimality system. We obtain the following adjoint system, which consists of the five ordinary differential equations with the final conditions, by applying Pontryagin's Maximum Principle,

$$
\begin{gathered}
\frac{d \lambda_{1}}{d t}=-\frac{\partial L}{\partial X}, \lambda_{1}\left(t_{f}\right)=0 \\
\vdots \\
\frac{d \lambda_{5}}{d t}=-\frac{\partial L}{\partial H}, \lambda_{5}\left(t_{f}\right)=0
\end{gathered}
$$

evaluated at the optimal control pair and corresponding states, which results in the stated adjoint system (6) and (7), [21].

Now consider the optimality conditions to derive the optimal controls. To illustrate the characterization of $u_{1}^{*}$ and $u_{2}^{*}$, we have

$$
\begin{aligned}
& \frac{\partial L}{\partial u_{1}}=B_{1} u_{1}(t)-\left(\lambda_{2}-\lambda_{1}\right) \omega X Y=0, \\
& \frac{\partial L}{\partial u_{2}}=B_{2} u_{2}(t)+\left(\lambda_{3}-\lambda_{5}\right) \beta_{2} S H=0
\end{aligned}
$$

at $u_{1}^{*}$ on the set $\left\{t \mid 0<u_{1}^{*}<1\right\}$ and $u_{2}^{*}$ on the set $\left\{t \mid 0<u_{1}^{*}<a\right\}$. Solving for the optimal controls on this set yields

$$
\begin{aligned}
& u_{1}^{*}(t)=\frac{1}{B_{1}}\left(\left(\lambda_{2}-\lambda_{1}\right) \omega X^{*} Y^{*}\right) \\
& \text { and } \\
& u_{2}^{*}(t)=\frac{1}{B_{2}}\left(\left(\lambda_{5}-\lambda_{3}\right) \beta_{2} S^{*} H^{*}\right) .
\end{aligned}
$$

Taking into account the bounds on $u_{1}^{*}$ and $u_{2}^{*}$, we obtain the characterizations of $u_{1}^{*}$ and $u_{2}^{*}$ in (8). 
Note that we obtain the uniqueness of the optimal control for small $t_{f}$ because of the a priori boundedness of the state and adjoint functions and the resulting Lipschitz structure of the ODEs. The unique optimal control pair is characterized in terms of the unique solution of the optimality system [10].

\section{Acknowledgements}

The authors would like to thank the editor and anonymous referee for very helpful suggestions and comments which improved the quality of this paper and study. Jung's work was supported by the Korea Research Foundation Grant funded by the Korean Government (MOEHRD, Basic Research Promotion Fund) (KRF-2007-531C00015) and Shingo's work was supported by Research Fellowships of the Japan Society for the Promotion of Science for Young Scientists.

\section{References}

[1] R. M. Anderson and R. M. May (1991) Infectious disease of humans: dynamics and control, Oxford University Press.

[2] R. Antia, R. R. Regoes, J. C. Koella, and C. T. Bergstrom (2003) The role of evolution in the emergence of infectious diseases, Nature, 426, 658-661.

[3] Centers for Disease Control and Prevention (2006) Avian influenza: current situation, http://www.cdc.gov/flu/avian/outbreaks/pdf/current.pdf, August 09.

[4] H. Chen, G. Deng, Z. Li, G. Tian, Y. Li, P. Jiao, L. Zhang, Z. Liu, R. G. Webster, and K. Yu (2004) The evolution of H5N1 influenza viruses in ducks in southern China, Proc. Nant. Acad. Sci. U.S.A., 101, 10452-10457.

[5] H. Chen, G. L. Smith, K. S. Li, J. Wang, X. H. Fan, J. M. Rayner, D. Vijaykrishna, J. X. Zhang, L. J. Zhang, C. T. Guo, C. L. Cheung, K. M. Xu, L. Duan, K. Huang, K. Qin, Y. H. Leung, W. L. Wu, H. R. Lu, Y. Chen, N. S. Xia, T. S. Naipospos, K. Y. Yuen, S. S. Hassan, S. Bahri, T. D. Nguyen, R. G. Webster, J. S. Peiris, and Y. Guan (2006) Establishment of multiple sublineages of H5N1 influenza virus in Asia: implications for pandemic control, Proc. Nant. Acad. Sci. U.S.A., 103, 2845-2850.

[6] G. Chowell, C. E. Ammon, N. W. Hengartner, and J. M. Hyman (2006) Transmission dynamics of the great influenza pandemic of 1918 in Geneva, Switzer- 
land: Assessing the effects of hypothetical interventions, J. Theor. Biol., 241, 193-204.

[7] G. Chowell and H. Nishiura (2008) Quantifying the transmission potential of pandemic influenza, Phy. Life Rev., 5, 50-77.

[8] N. M. Ferguson, D. A. T. Cummings, S. Cauchemez, C. Fraser, S. Riley, A. Meeyai, S. Iamsirithaworn and D. S. Burke (2005) Strategies for containing an emerging influenza pandemic in Southeast Asia, Nature, 437, 209-214.

[9] N. M. Ferguson, D. A. T. Cummings, C. Fraser, J. C. Cajka, P. C. Cooley and D. S. Burke (2006) Strategies for mitigating an influenza pandemic, Nature, 442, 448-452.

[10] K. R. Fister, S. Lenhart, and J. S. McNally (1998) Optimizing chemotherapy in an HIV model, E. J. Diff. Equ., 32, 1-12.

[11] W. H. Fleming and R. W. Rishel (1975) Deterministic and stochastic optimal control, Springer Verlag, New York.

[12] A. Gambotto, S. M Barratt-Boyes, M. D de Jong, G. Neumann, and Y. Kawaoka (2008) Human infection with highly pathogenic H5N1 influenza virus, Lancet, 371, 1464-1475.

[13] M. Gilbert, X. Xiao, J. Domenech, J. Lubroth, V. Martin, and J. Slingenbergh (2006) Anatidae migration in the Western Palearctic and spread of highly pathogenic avian influenza H5N1 virus, Emer. Infec. Dise., 12, 1650-1656.

[14] W. Hogg, D. Gray, P. Huston, and W. Zhang (2007) The costs of preventing the spread of respiratory infection in family physician offices: a threshold analysis, BMC Health Serv. Res., 7, 181.

[15] S. Iwami, Y. Takeuchi, and X. Liu (2007) Avian-human influenza epidemic model, Math. Bios., 207, 1-25.

[16] S. Iwami, Y. Takeuchi, A. Korobeinikov, and X. Liu (2008) Prevention of avian influenza epidemic: what policy should we choose?, J. Theor. Biol., 252, 732741.

[17] S. Iwami, Y. Takeuchi, and X. Liu (2009) Avian flu pandemic: can we prevent it?, J. Theor. Biol., 257, 181-190. 
[18] E. Jung, S. Lenhart, and Z. Feng (2002) Optimal control of treatments in a two strain tuberculosis model, Dis. Cont. Dyn. Sys. B, 2, 473-482.

[19] E. Jung, S. Lenhart, V. Protopopescu, and C. Babbs (2005) Optimal control theory applied to a difference equation model for cardiopulmonary resuscitation, Math. Meth. Mod. Appl. Sci., 15, 1519-1532.

[20] E. Jung, S. Lenhart, V. Protopopescu, and C. Babbs (2006) Optimal strategy for cardiopulmonary resuscitation with continuous chest compression, Acad. Emer. Med., 7, 715-721.

[21] M. I. Kamien and N. L. Schwarz (1991) Dynamic optimization: the calculus of variations and optimal control, North Holland, Amsterdam.

[22] D. Kirschner, S. Lenhart, and S. Serbin (1997) Optimal control of the chemotherapy of HIV, J. Math. Biol., 35, 775-792.

[23] T. T. Lam, C. C. Hon, O. G. Pybus, S. L. Kosakovsky Pond, R. T. Wong, C. W. Yip, F. Zeng, and F. C. Leung (2007) Evolutionary and transmission dynamics of reassortant H5N1 influenza virus in Indonesia, PLoS Pathog., 4(8), e1000130.

[24] S. Lenhart, V. Protopopescu, E. Jung, and C. Babbs (2005) Optimal control for a standard CPR Model, Non. Anal., 63, 1391-1397.

[25] S. Lenhart and J. T. Workman (2007) Optimal control applied to biological models, Chapman and Hall/CRC Mathematical and Computational Biology Series.

[26] N. T. Liem, World Health Organization International Avian Influenza Investigation Team, Vietnam and W. Lim (2005) Lack of H5N1 avian influenza transmission to hospital employees, Hanoi, 2004 Emer. Infec. Dise., 1, 210-215.

[27] J. D. Mathews, C. T. McCaw, J. McVernon, E. S. McBryde, and J. M. McCaw (2007) A biological model for influenza transmission: pandemic planning implications of asymptomatic infection and immunity, PLoS ONE, 2(11), e1220.

[28] A. McLeod, J. Rushton, A. Riviere-Cinnamond, B. Brandenburg, J. Hinrichs and L. Loth (2007) Economic issues in vaccination against highly pathogenic avian influenza in developing countries, Dev. Biol. (Basel), 130, 63-72. 
[29] A. L. Menach, E. Vergu, R. F. Grais, D. L. Smith, and A. Flahault (2006) Key strategies for reducing spread of avian influenza among commercial poultry holdings: lessons for transmission to humans, Proc. R. Soc. Lond B, 273, 24672475 .

[30] C. E. Mills, J. M. Robins, and M. Lipsitch (2004) Transmissibility of 1918 pandemic influenza, Nature, 432, 904-906.

[31] D. Normile (2006) Avian influenza. WHO proposes plan to stop pandemic in its tracks, Science, 311, 315-316.

[32] The New York Times (2006) In the Nile delta, bird flu preys on ignorance and poverty, April 13.

[33] M. Peyre, G. Fusheng, S. Desvaux and F. Roger (2008) Avian influenza vaccines: a practical review in relation to their application in the field with a focus on the Asian experience, Epidemiol. Infect., 14, 1-21.

[34] L. S. Pontryagin, V. G. Boltyanskii, R. V. Gamkrelidze, and E. F. Mishchenko (1962) The mathematical theory of optimal processes, Wiley, New York.

[35] G. A. Poland, R. M. Jacobson, and P. V. Targonski (2007) Avian and pandemic influenza: an overview, Vaccine, 25, 3057-3061.

[36] R. R. Regoes and S. Bonhoeffer (2006) Emergence of drug-resistant influenza virus: population dynamical considerations, Nature, 312, 389-391.

[37] J. Rushton, R. Viscarra, E. Guerne Bleich and A. McLeod (2005) Impact of avian influenza outbreaks in the poultry sectors of five South East Asian countries (Cambodia Indonesia Lao PDR Thailand Viet Nam) outbreak costs responses and potential long term control, World's Poultry Science Journal, 61, 491-514.

[38] B. Sander, A. Nizam, L. P. Garrison, M. J. Postma, M. E. Halloran, and I. M. Longini (2008) Economic evaluation of influenza pandemic mitigation strategies in the United States using a stochastic microsimulation transmission model, Value. Health, Jul. 30.

[39] S. Sethi and G. L. Thompson (2000) Optimal control theory: applications to management science and economics, Kluwer Academic, Boston.

[40] N. Skeik and F. I. Jabr (2008) Influenza viruses and the evolution of avian influenza virus H5N1, Int. J. Infec. Dise., 12, 233-238. 
[41] A. Stegeman, A. Bouma, A. R. W. Elbers, M. C. M. de Jong, G. Nodelijk, F. de Klerk, G. Koch, and M. J. van Boven (2004) Avian influenza A virus (H7N7) epidemic in The Netherlands in 2003: course of the epidemic and effectiveness of control measures, J. Infect. Dis., 190, 2088-2095.

[42] J. K. Taubenberger, A. H. Reid, and T. G. Fanning (2005) Capturing a killer flu virus, Sci. Amer., January.

[43] T. Tiensin, P. Chaitaweesub, T. Songserm, A. Chaisingh, W. Hoonsuwan, C. Buranathai, T. Parakamawongsa, S. Premashthira, A. Amonsin, M. Gilbert, M. Nielen, and A. Stegeman (2005) Highly pathogenic avian influenza H5N1, Thailand, 2004, Emerg. Infect. Dis., 11, 1664-1672.

[44] T. Tiensin, M. Nielen, H. Vernooij, T. Songserm, W. Kalpravidh, S. Chotiprasatintara, A. Chaisingh, S. Wongkasemjit, K. Chanachai, W. Thanapongtham, T. Srisuvan, and A. Stegeman (2007) Transmission of the highly pathogenic avian influenza virus H5N1 within flocks during the 2004 epidemic in Thailand, J. Infec. Dis., 196, 1679-1684.

[45] T. van den Berg and P. Houdart (2008) Avian influenza outbreak management: action at time of confirmation, depopulation and disposal methods; the 'Belgian experience' during the H7N7 highly pathogenic avian influenza epidemic in 2003, Zoono. Pub. Health, 55, 54-64.

[46] R. G. Webster, M. Peiris, H. Chen, and Y. Guan (2006) H5N1 Outbreaks and enzootic influenza, Emer. Infec. Dise., 12, 3-8.

[47] L. F. White and M. Pagano (2008) Transmissibility of the influenza virus in the 1918 pandemic, PLoS ONE, 3(1), e1498.

[48] The World Health Organization (2005) Global influenza preparedness plan, http:// www.who.int/csr/resources/publications/influenza/WHOCDSCSRGIP20055.pdf.

[49] The world Health Organization (2006) Avian influenza: significance of mutations in the H5N1 virus, http://www.who.int/csr/20060220/en/index.html, February 20.

[50] The Writing Committee of the World Health Organization (WHO) Consultation on Human Influenza A/H5 (2006) Avian influenza A (H5N1) infection in humans, N. Engl. J. Med., 353, 1374-1385. 
[51] K. S. Yee, T. E. Carpenter, and C. J. Cardona (2008) Epidemiology of H5N1 avian influenza, Comp. Immun. Microbiol. Infect. Dis., In Press. 

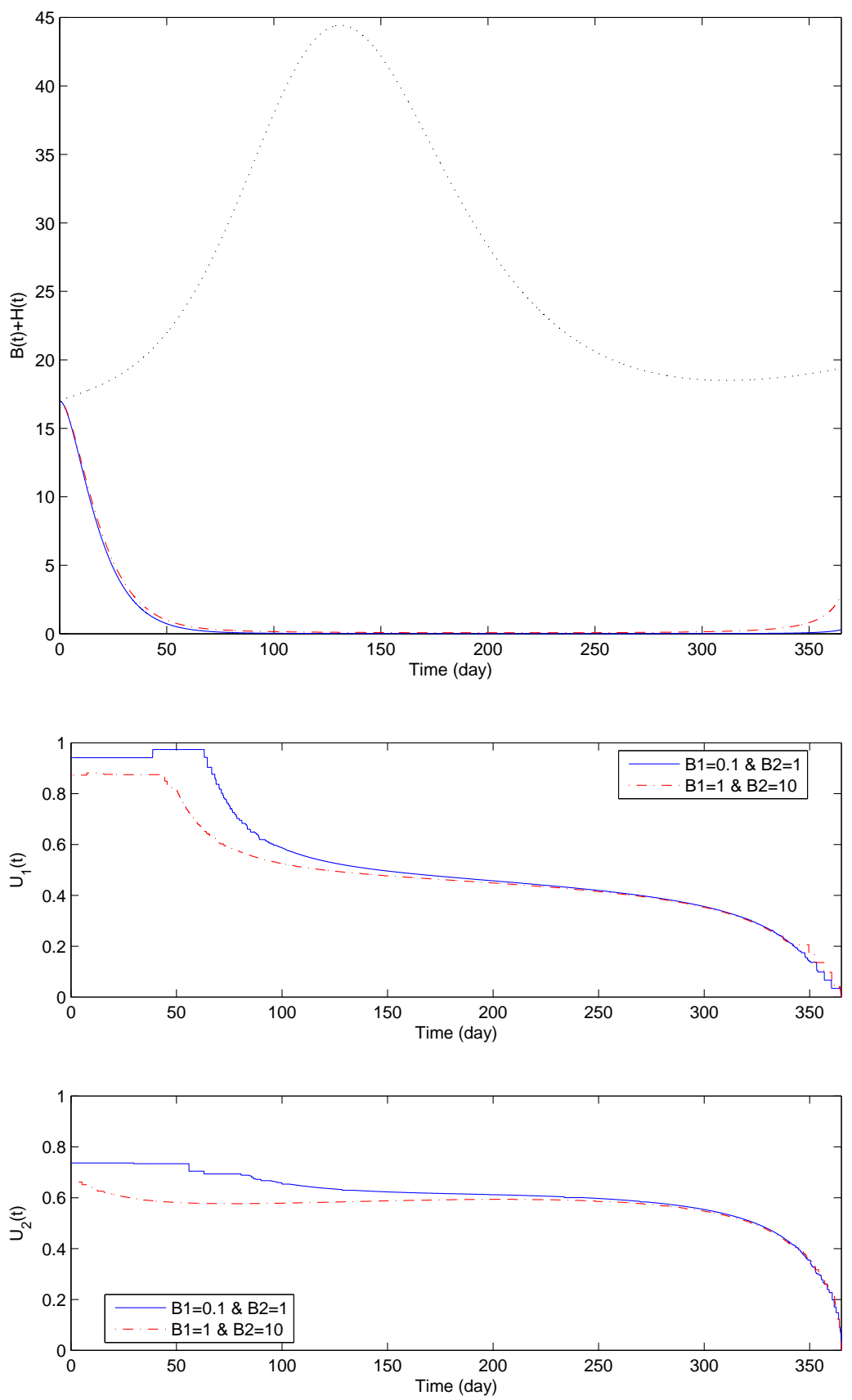

Figure 1: Time-course of disease spread with optimal prevention policies for 365 days after the emergence of mutant avian influenza virus in the lower virulence case: the top figure shows that the epidemic curves of total infected humans without (dashed black curve) and with (colored curves: the blue and red curves correspond to $\left(B_{1}, B_{2}\right)=(0.1,1)$ and $(1,10)$, respectively) optimal prevention policies. The bottom two figures show the time-dependent optimal strategy of $u_{1}$ and $u_{2}$. The optimal strategy dramatically reduces the epidemic curve and shows that the quarantine policy is more important because the long strong effort of the quarantine policy $u_{2}^{*}(t)$ is needed over the simulated time. 

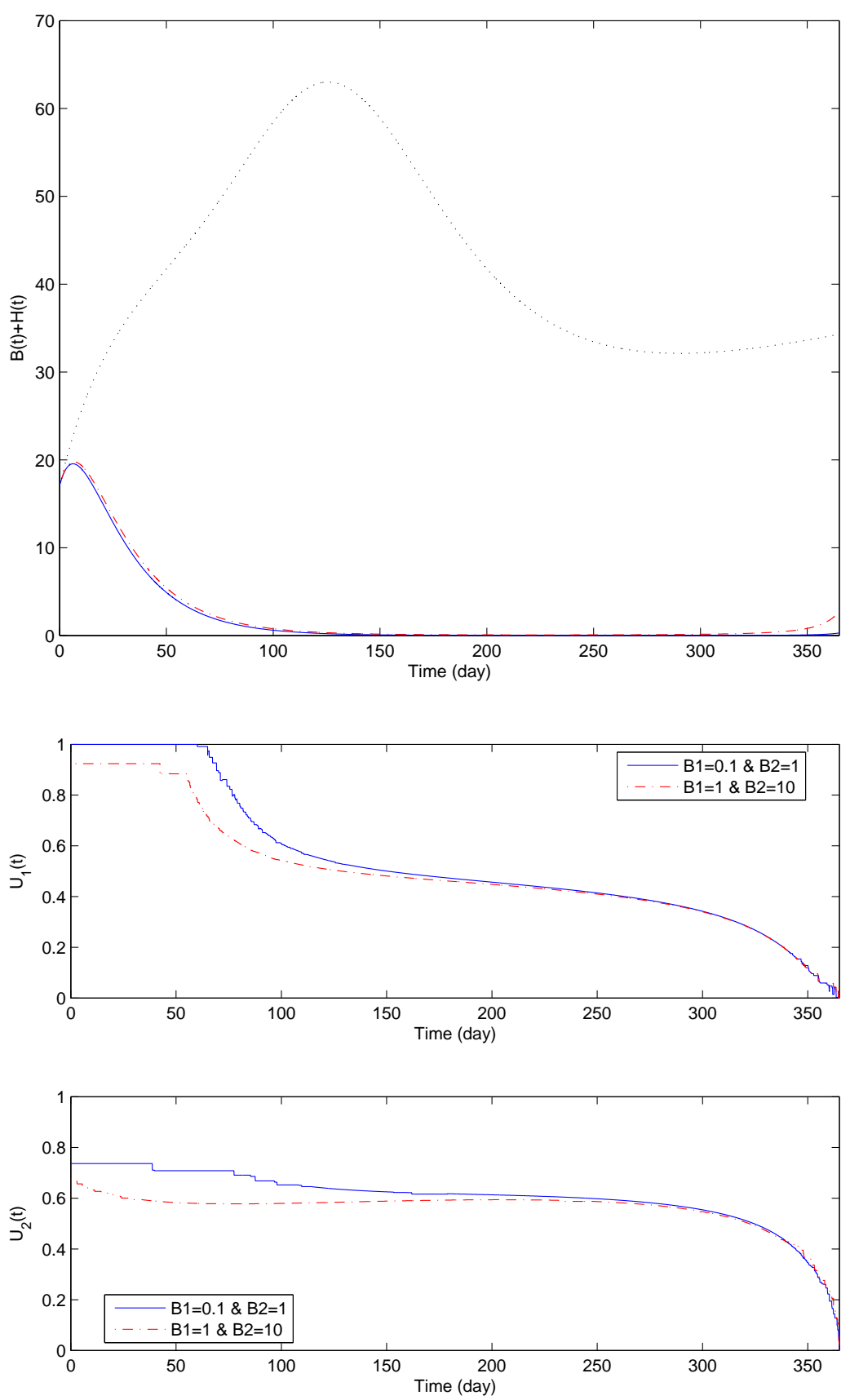

Figure 2: Time-course of disease spread with optimal prevention policies for 365 days after the emergence of mutant avian influenza virus in the higher virulence case: the top figure shows that the epidemic curves of total infected humans without (dashed black curve) and with (colored curves: the blue and red curves correspond to $\left(B_{1}, B_{2}\right)=(0.1,1)$ and $(1,10)$, respectively) optimal prevention policies. The bottom two figures show the time-dependent optimal strategy of $u_{1}$ and $u_{2}$. The optimal strategy dramatically reduces the epidemic curve and shows that the quarantine policy is more important because the long strong effort of the quarantine policy $u_{2}^{*}(t)$ is needed over the simulated time. 
The cost of elimination and quarantine policies

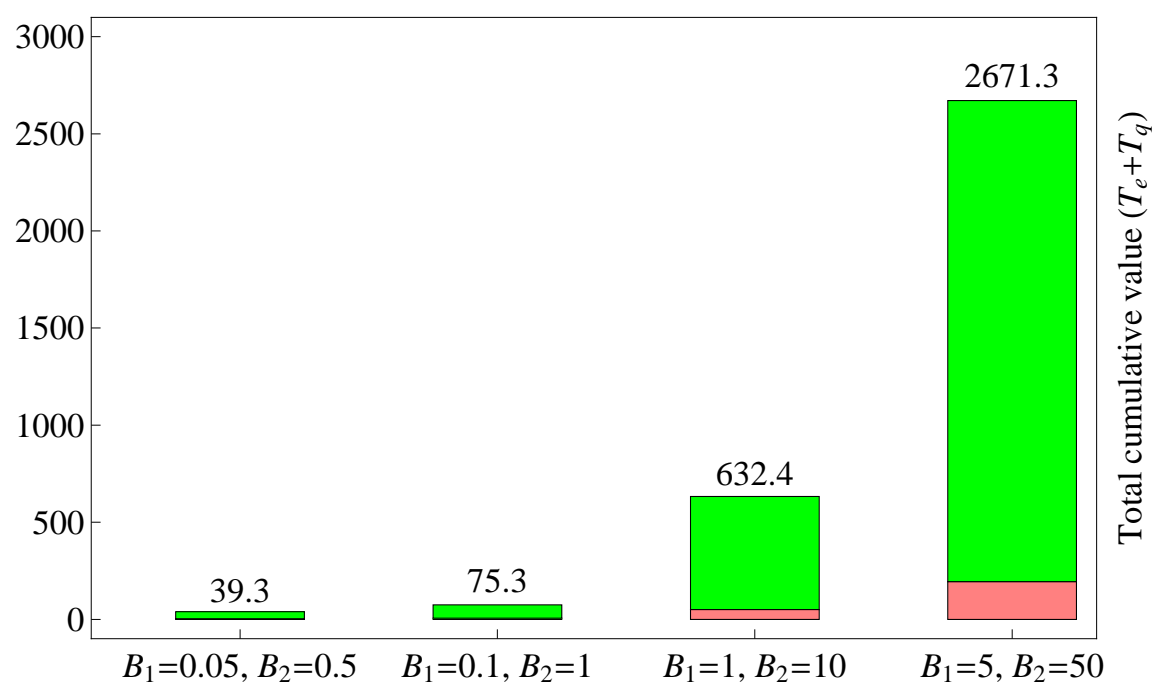

The ratio of cost for elimination and quarantine policies

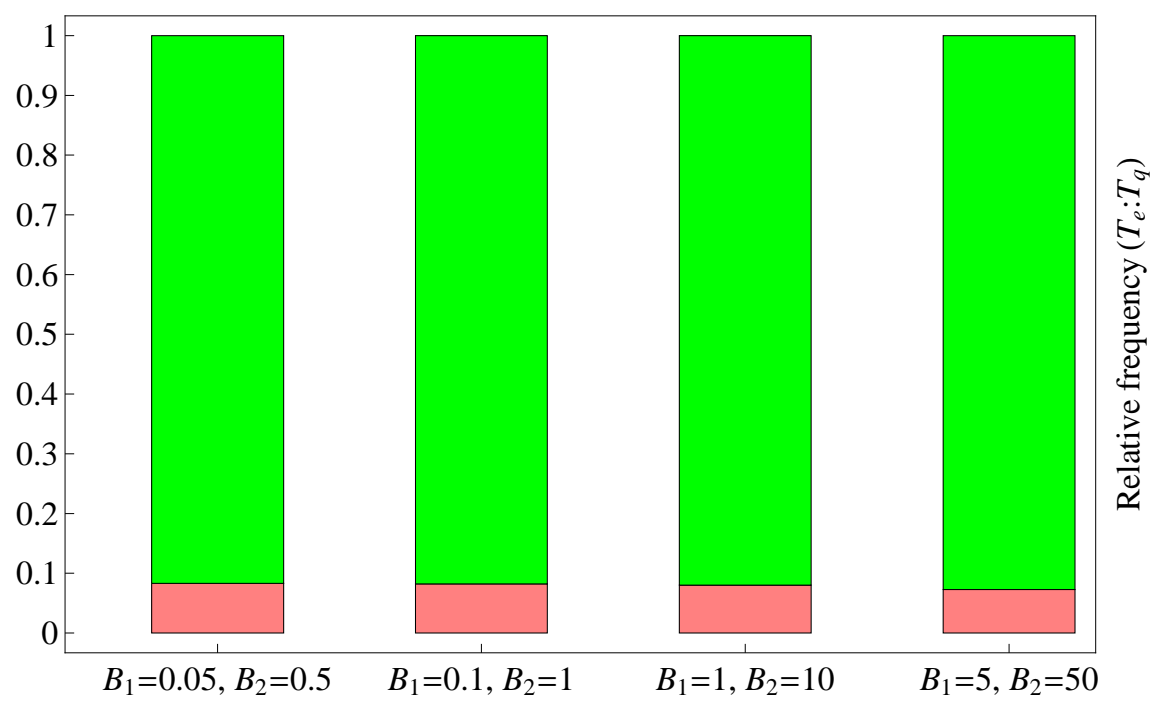

Figure 3: Cumulative execution cost of elimination and quarantine policies: we calculate each cumulative execution cost for the case $\left(B_{1}, B_{2}\right)=(0.05,0.5),(0.1,1),(1,10)$ and $(5,50)$ in the lower virulence case. The top and bottom figures, respectively, represent the total cumulative cost $\left(T_{e}+T_{q}\right)$ and the relative frequency of each cumulative execution cost $\left(T_{e}: T_{q}\right)$. Here the pink and green bars correspond to the cumulative cost of the elimination and quarantine policies, respectively. The optimal strategy shows that the change of the unit execution cost does affect the total cumulative cost but does not affect the relative frequency of each cumulative execution cost. Thus, the relative cost of using the quarantine policy is much expensive than one of using the elimination policy. 


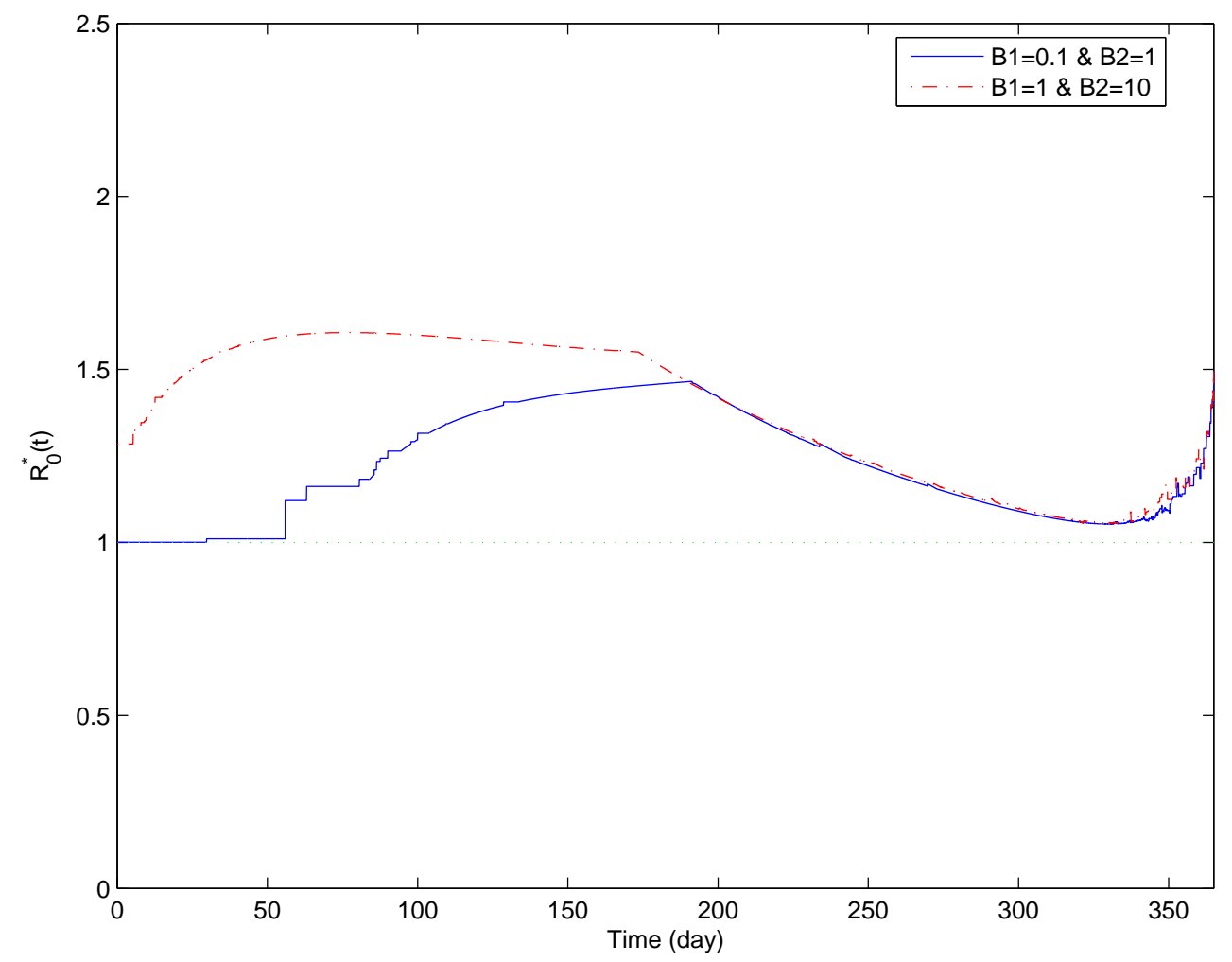

Figure 4: Invadability of mutant avian influenza: we calculate the invadability measure of the mutant strain $\bar{R}_{0}^{*}(t)$ during the optimal prevention policies for the unit execution cost $\left(B_{1}, B_{2}\right)=(0.1,1)$ and $(1,10)$ in the lower virulence case. The optimal strategy suppresses the spread of the mutant strain at the beginning. In general, as the unit execution cost increases, the invadability measure increases over the simulated time. Furthermore, interestingly, the invadability measure gradually increases at the beginning of the optimal strategy and has a peak in both cases. The optimal strategy to reduce the number of total infected humans might increase a chance of invadability of mutant avian influenza. That is, the optimal strategy is no longer optimum in terms of prevention of invadability of mutant avian influenza. 